\title{
Fueling Clean Household Environments
}

\author{
Kalpana Balakrishnan \\ ${ }^{1}$ SRU-ICMR Center for Advanced Research on Air Quality, Climate and Health, Sri Ramachandra Institute of Higher Education and Research \\ (Deemed to be University), Chennai, India
}

The World Health Organization (WHO) defines household air pollution (HAP) as air pollution generated by household fuel combustion, leading to indoor air pollution and contributing to ambient air pollution (WHO 2014). From the first study some 30 years ago, which reported rural Indian women's personal exposure measurements to particulate matter from using biomass cookstoves to the hundreds of studies that now present HAP exposure measurements, these have collectively and unequivocally shown air pollution levels in homes that burn solid fuels to be unacceptably high, exposing women, men and children to pollutant concentrations well in excess of WHO air quality guidelines. The health burden attributable to HAP is massive, with WHO estimating 2.6-3.8 million premature deaths resulting from child pneumonia, ischemic heart disease, stroke, chronic obstructive lung disease and lung cancer, mostly among the rural poor of low- and middleincome countries (LMICs). The list of diseases impacted by HAP continues to expand, including early data for diabetes and cognitive effects, and now includes nearly every health end point shown to be previously associated with active and passive tobacco smoking.

While images of poor rural women engulfed in a plume of smoke from their cookstoves as they prepare the everyday family meal abound in scientific and development literature, the artwork presented in the cover is unique in several ways. It serves as a poignant reminder for the global scientific community to rally around a health issue that

Published online: October 11, 2018

Correspondence to: Kalpana Balakrishnan,e-mail: kalpanasrmc@ehe.org.in continues to be an everyday reality for 3 billion people in low- and middle-income countries. It addresses the complexity of the 'seemingly simple' task of making a meal for a family. While their urban counterparts have multiple clean and convenient technologies to choose from, rural women are challenged by more than just air pollution as they juggle the numerous cooking-related tasks on inefficient cookstoves. The sheer physical hardship of preparing the meal is unmistakable, yet the meal is what makes her house a home!

While evidence on the impacts of solid fuel use has been available for nearly two decades, the scientific understanding of how to ameliorate the situation has undergone a sea change in the last decade. In 2015, Dr. Kirk R. Smith provided an eloquent description of new paradigms for accelerating the transition to cleaner cooking (Smith 2015). Much scientific evidence has accumulated to lend additional credence to the proposed paradigms, which emphasize the cleanest possible fuels. Clean fuels (e.g., liquified petroleum gas or LPG) are the only technologies that will enable households to meet WHO air quality guidelines. Virtually, every survey that has assessed user perceptions for fuel choices, across more than a dozen countries, reports a strong preference for LPG over biomass fuel. Although multiple reasons are cited as barriers for sustained adoption of clean fuels, economic barriers that preclude use of clean fuels remain the overwhelming reason behind inadequate levels of use among poor communities.

The artwork provides a beautiful imagery of igniting the flame on a clean stove that perhaps symbolizes the 
innate desire of every poor household to dispel the darkness of poverty. Access to LPG can open the door for the poor to fulfill their aspirational goals for their own household. While access to clean fuels is not sufficient to reduce HAP exposures, it is a necessary first step on the journey to a complete transition to clean household energy, along a trajectory of reduced exposures (as shown by the graph on the cover). Indeed, the impact of clean fuels on time savings (resulting from elimination or reduction in time for fuel collection as well as faster cooking), cleaner kitchens, improved air quality and health symptoms are perceived almost instantaneously wherever LPG has been introduced at the community or programmatic levels (across countries of South Asia, Africa or South America). This is in sharp contrast to the 'poor' or at best 'mixed' feedback obtained from numerous 'improved biomass cookstove' studies from the same countries that have reported limited use and insignificant health relevant reductions in HAP exposures. Indeed, the astounding success of the massive roll out of the LPG program in India (termed the Pradhan Mantri Ujjwala Yojana) that seeks to provide free 'connections' (a term that denotes improved access to an LPG distribution network) to 80 million households by 2019 bears testimony for overwhelming community preference for clean fuels.

The newest scientific evidence gathered from personal exposure and rural ambient monitoring studies and assessment of contributions from residential solid fuel use to ambient concentrations, however, point to the need to do more than just increase access. Without a critical mass of households that transition completely, no one stands to gain on health. Indeed, the WHO definition of household air pollution and the accompanying air quality guidelines provide an overarching framework for designing and implementing HAP interventions. The guidelines do not differentiate between indoor vs outdoor or rural vs urban environments for attainment of recommended standards of air quality. It has now become clear that it will be virtually impossible to meet the ambient air quality guidelines in communities or countries with significant solid fuel use without the complete transition to clean fuels. The scien- tific foresight used in formulating the guidelines is now backed by an emerging and expanding pool of evidence.

There is now a remarkable confluence of favorable circumstances to push the case for clean fuels in LMICs. Quantitative evidence on HAP exposure reductions, associated with LPG use and user preferences for LPG as a clean fuel choice, is now available from numerous LMICs. The pool of evidence on health effects draws heavily on the literature from LMICs despite the relative paucity of longterm mortality studies. Programmatic infrastructure to supply and sustain LPG use is in place in many LMICs, most notably in populous countries such as India. Emissions from residential cooking are identified as a major contributor to the burden of disease attributable to both ambient and household air pollution. Ongoing and newly launched randomized control trials (that include exposureresponse analyses) are expected to provide considerable additional evidence for health outcomes for vulnerable groups (including pregnant women and infants). Several insights for implementation of LPG programs are also expected to be gleaned from these studies. Meanwhile, much can be gained now, by pushing the clean cooking agenda within the health, energy and environment sectors. More importantly, much health would be lost for the rural poor if imminent actions are not facilitated at national, regional and global levels.

Fueling clean household environments is within our reach. The art on the cover and collection of articles in the issue can be yet another vehicle to bring concerted actions for the health of our women, men and children at risk from HAP exposures.

\section{REFERENCES}

Smith KR (2015) Changing paradigms in clean cooking. EcoHealth 12(1):196-199. https://doi.org/10.1007/s10393-0151020-9

World Health Organisation (WHO) (2014) WHO guidelines for indoor air quality: household fuel combustion, Geneva, Switzerland: World Health Organisation 